\title{
REMOÇÃO DO CORANTE DE URUCUM PELA ENZIMA Horseradish peroxidase
}

\author{
R. E. FRANZOI JR ${ }^{1}$, S. FARIAS ${ }^{1}$, M. A. BOTH ${ }^{1}$, D. TODESCATO ${ }^{1}$, S. M. A. GUELLI U.
} SOUZA $^{1}$, A. A. ULSON DE SOUZA ${ }^{1}$

${ }^{1}$ Universidade Federal de Santa Catarina, Departamento de Engenharia Química e Engenharia de Alimentos

E-mail para contato: simonefarias2005@yahoo.com.br, augusto@enq.ufsc.br

RESUMO: Atualmente cresce a demanda pelos corantes naturais na substituição dos corantes sintéticos, por estes serem considerados mais seguros para o consumidor. $\mathrm{O}$ uso de enzimas para a degradação dos corantes residuais dos efluentes têxteis é considerado um processo inovador e ecologicamente correto. Assim, o objetivo deste trabalho é estudar a remoção do corante natural de urucum utilizando a enzima Horseradish peroxidase. $\mathrm{O}$ efeito dos parâmetros relevantes do processo como concentração de corante, enzima e peróxido de hidrogênio e $\mathrm{pH}$ foram determinados. A metodologia da superfície de resposta foi utilizada na otimização do processo de degradação do corante. As faixas ótimas de concentração de enzima e peróxido de hidrogênio foram determinadas para degradar uma solução de $550-850 \mathrm{mg} / \mathrm{L}$ de corante de urucum (bixina), na faixa de $\mathrm{pH} 4,5-7,0$. Foi utilizado um limite de confiança de $95 \%$ e obtido um ajuste ao modelo de $70 \%$.

\section{INTRODUÇÃO}

Atualmente em indústrias de todo mundo os corantes mais utilizados são os corantes sintéticos, principalmente pela solidez ao tingimento e por proporcionar técnicas mais reprodutíveis (HADDAR et al., 2014). Porém, quando descartados no meio ambiente esses corantes possuem a capacidade de alterar ciclos biológicos e podem resultar em um grande impacto ambiental, por suas propriedades mutagênicas e carcinogênicas (NGUEN; JUANG, 2013; KUMAR et al., 2012; KARIM; ADNAN; HUSAIN, 2012).

No decorrer dos últimos anos vem crescendo a preocupação ambiental pela utilização de produtos mais limpos, biodegradáveis e não recalcitrantes nos diversos setores industriais. Os corantes naturais derivados de plantas, insetos/animais são de fontes renováveis e com mínimo impacto ambiental, além de serem utilizados como corantes têxteis também são muito 
utilizados nas indústrias de alimentos e cosméticos (MOHAMMAD; UL-ISLAM; MOHAMMAD, 2013).

Deste ponto de vista a degradação enzimática surge como uma alternativa ecologicamente correta aos métodos tradicionais de remoção de cor de efluentes têxteis. As enzimas são biodegradáveis e são muito eficientes, principalmente devido à capacidade de converter estruturas químicas complexas sob condições suaves de processo (SILVA et al., 2013). Enzimas das classes das oxidorredutases tais como peroxidase e lacase têm mostrado capacidade para degradar uma variedade de compostos orgânicos como os corantes. Estas enzimas podem catalisar a degradação de corantes, tanto por precipitação como pela quebra do anel aromático (SILVA et al., 2012).

Peroxidases são enzimas produzidas por um grande número de microrganismos e plantas que catalisam uma variedade de reações, requerendo a presença de peróxido de hidrogênio para à ação da mesma. Entre as peroxidases a mais conhecida é a Horseradish peroxidase, que tem sido utilizada na remoção de vários contaminantes aromáticos, principalmente porque ela mantém sua atividade sobre uma ampla faixa de $\mathrm{pH}$ e temperatura. Uma vez ativada pelo peróxido esta enzima atua sobre uma variedade de compostos tóxicos como fenóis, corantes, anilinas, entre outros (KARAM; NICELL, 1997; BUCHANAN; NICELL, 1998).

Este estudo avalia o uso da enzima Horseradish peroxidase para catalisar a degradação do corante natural de urucum através da metodologia da superfície de resposta. Ainda existem poucos trabalhos na literatura sobre a degradação de corantes naturais, sendo relevante, portanto, avaliação dos principais fatores que influenciam esse processo.

\section{MATERIAIS E MÉTODOS}

\subsection{Corante}

O corante utilizado foi o corante natural de urucum, Eq. 1 (a), que em solução alcalina, passa a ter o elemento ativo norbixina, Eq. 1 (b). A solução de corante contém hidróxido de potássio, água e urucum. As estrutura da bixina e norbixina são apresentadas na Figura 1.<smiles>COC(=O)/C=C/C(C)=C\C=C\C(C)=C\C=C\C=C(C)\C=C\C=C(C)\C=C\C(=O)O</smiles><smiles>CC(/C=C/C=C(C)/C=C/C(=O)O)=C\C=C\C=C(C)\C=C\C=C(C)\C=C\C(=O)O</smiles>

Figura 1- Estrutura da (a) bixina e (b) norbixina 


\subsection{Atividade enzimática}

A atividade inicial do extrato enzimático da Horseradish peroxidase doada pela empresa Toyobo foi determinada segundo a metodologia modificada de Wu et al. (1997). Para a determinação da atividade foram utilizados $0,255 \mathrm{~mL}$ de solução de ABTS $20 \mathrm{mM}$; $0,255 \mathrm{~mL}$ de peróxido de hidrogênio $\left(\mathrm{H}_{2} \mathrm{O}_{2}\right) 10 \mathrm{mM} ; 0,300 \mathrm{~mL}$ de extrato enzimático $1 \mathrm{mM}$; $2,19 \mathrm{~mL}$ de tampão fosfato $\mathrm{pH} 6\left(0,05 \mathrm{molL}^{-1}\right)$. Todas as soluções foram preparadas em tampão fosfato $\mathrm{pH}$ 6. A reação foi mantida a $30^{\circ} \mathrm{C}$ em banho termostatizado por $10 \mathrm{~min}$ e em seguida acompanhada durante $5 \mathrm{~min}$, em um espectrofotômetro a $420 \mathrm{~nm}$ utilizando um coeficiente de extinção molar de $\left(\varepsilon_{420 \mathrm{~nm}}=3,6 \times 10^{4} \mathrm{M}^{-1} . \mathrm{cm}^{-1}\right)$. A atividade enzimática (A) foi calculada conforme a Equação 1. Onde: $A=$ Atividade enzimática, $\Delta_{\mathrm{Abs}}=$ Absorvância ${ }_{\text {final }}-$ Absorvância inicial, $V_{\text {total }}=$ Volume total de solução na cubeta em $\mathrm{mL}, \mathrm{V}$ Extrato $=$ Volume de extrato enzimático utilizado na reação $\mathrm{em} \mathrm{mL}, \mathrm{t}=$ tempo de reação em minutos.

$$
\mathrm{A}(\mathrm{U} / \mathrm{mL})=\frac{\Delta_{\text {Abs. }} . \mathrm{V}_{\text {total. }} 10^{6}}{\varepsilon_{\mathrm{ABTS} . \text { Vextrato. }} \mathrm{t}}
$$

\subsection{Degradação Enzimática}

As reações de degradação enzimática foram conduzidas conforme metodologia modificada de Silva et al., (2012). Foram utilizados $0,4 \mathrm{~mL}$ de solução de $\mathrm{H}_{2} \mathrm{O}_{2}, 0,1 \mathrm{~mL}$ de extrato enzimático, $1,5 \mathrm{~mL}$ de corante e tampão citrato ou fosfato $0,05 \mathrm{~mol} / \mathrm{L}(1,2 \mathrm{~mL})$. As soluções de $\mathrm{H}_{2} \mathrm{O}_{2}$ e de extrato enzimático foram preparadas em tampão nos diferentes $\mathrm{pHs}$ estudados, a solução de corante foi preparada em água destilada. Nos controles reacionais foram empregados todos os reagentes com exceção do $\mathrm{H}_{2} \mathrm{O}_{2}$ que foi substituído por tampão. A absorvância foi medida no comprimento de onda do urucum $380 \mathrm{~nm}$. A porcentagem de degradação (D) foi calculada pela equação 2 .

$$
\frac{D(\%)=A b s_{\text {inicial }}-A b s_{\text {final }}}{A b s_{\text {inicial }}} .100
$$

\subsection{Planejamento experimental}

A degradação do corante de urucum foi realizada utilizando um planejamento fatorial estrela $2^{4}$ com triplicata do ponto central totalizando 27 ensaios, realizados de maneira aleatória. As variáveis analisadas foram concentração de corante, enzima, peróxido de hidrogênio e pH e como resposta foi obtida a descoloração do corante de urucum. O software utilizado foi o Statistic versão 10. As variáveis utilizadas no planejamento são apresentadas na Tabela 1. 
Tabela 1- Variáveis e níveis de estudo utilizados no planejamento experimental

\begin{tabular}{cccccc}
\hline Variáveis & \multicolumn{5}{c}{ Níveis de estudo } \\
\hline $\begin{array}{c}\text { Concentração de } \\
\text { Enzima U/mL }\end{array}$ & 576,9 & 1153,9 & 1730,8 & 2307,7 & 2884,6 \\
$\begin{array}{c}\text { Concentração de } \\
\quad \mathbf{H}_{2} \mathbf{O}_{2} \mu \mathrm{M}\end{array}$ & 150,0 & 200,0 & 250,0 & 300,0 & 350,0 \\
$\begin{array}{c}\text { Concentração de } \\
\text { corante } \mathrm{mg} / \mathrm{L} \\
\text { pH }\end{array}$ & 200,0 & 400,0 & 600,0 & 800,0 & 1000,0 \\
\hline
\end{tabular}

\section{RESULTADOS E DISCUSSÃO}

A metodologia da superfície de resposta é uma técnica de otimização de experimentos baseada em planejamentos fatoriais que pode aumentar o rendimento, reduzir a variabilidade do processo e diminuir os custos. Através da análise de todas as variáveis é possível identificar aquelas que mais influenciam no experimento e as interações entre todas as variáveis. A precisão dos modelos é avaliada pelo coeficiente de determinação $\mathrm{R}^{2}$ (TAVARES et al., 2009; BALAN; SATHISHKUMAR; PALVANANN, 2012).

O planejamento realizado foi um planejamento fatorial do tipo estrela com 4 variáveis (concentração de enzima, peróxido, corante e $\mathrm{pH}$ ), neste planejamento foram realizados $2^{4}$ ensaios para investigação de um modelo linear, triplicata do ponto central e mais 8 pontos axiais distribuídos rotacionalmente a uma distância do ponto central, onde +2 e -2 para investigação do modelo quadrático. Os experimentos foram realizados de forma aleatória e a resposta obtida, a degradação do corante e apresentada na Tabela 2.

Os resultados mostraram que quando foi utilizada a enzima Horseradish peroxidase para degradar corante natural de urucum foram obtidas degradações de $8-44 \%$. As descolorações mínima e máxima foram obtidas com 1153,9 U/mL de enzima, $800 \mathrm{mg} / \mathrm{L}$ de corante e $\mathrm{pH}$ 7,0. Pode-se observar que o peróxido de hidrogênio foi determinante na descoloração, onde na concentração de $200 \mathrm{mM}$ de peróxido de hidrogênio a enzima degradou $8 \%$ do corante e quando e na concentração de $300 \mathrm{mM}$ foi obtida uma degradação de $44 \%$.

As enzimas peroxidases catalisam uma variedade de reações, porém todas requerem a presença de peróxido de hidrogênio para ativar a enzima. Vários estudos relatam a influências de fatores como peróxido de hidrogênio, concentração de corante e enzima. Silva et al., (2012) realizaram a descoloração dos corantes azo remazol laranja brilhante $3 R$ e vermelho remazol ultra, foram obtidas degradações de $9 \%$ e 5,5 \% respectivamente, esta baixa descoloração pode estar relacionada a fatores como concentração de enzima, peróxido de hidrogênio ou concentração de corante. 
Tabela 2- Planejamento experimental da descoloração enzimática do corante de urucum

\begin{tabular}{cccccc}
\hline Ensaio & $\begin{array}{c}\text { Enzima } \\
\text { U/mL }\end{array}$ & $\begin{array}{c}\mathbf{H}_{\mathbf{2}} \mathbf{O}_{\mathbf{2}} \\
\mathbf{m M}\end{array}$ & $\begin{array}{c}\text { Corante } \\
\mathbf{m g} / \mathbf{L}\end{array}$ & $\mathbf{p H}$ & $\begin{array}{c}\text { Descoloração } \\
(\mathbf{\%})\end{array}$ \\
\hline $\mathbf{1}$ & 1153,9 & 200,0 & 40,0 & 4,0 & 26,0 \\
$\mathbf{2}$ & 1153,9 & 200,0 & 40,0 & 7,0 & 9,0 \\
$\mathbf{3}$ & 1153,9 & 200,0 & 80,0 & 4,0 & 23,0 \\
$\mathbf{4}$ & 1153,9 & 200,0 & 80,0 & 7,0 & 8,0 \\
$\mathbf{5}$ & 1153,9 & 300,0 & 40,0 & 4,0 & 26,0 \\
$\mathbf{6}$ & 1153,9 & 300,0 & 40,0 & 7,0 & 28,0 \\
$\mathbf{7}$ & 1153,9 & 300,0 & 80,0 & 4,0 & 21,0 \\
$\mathbf{8}$ & 1153,9 & 300,0 & 80,0 & 7,0 & 44,0 \\
$\mathbf{9}$ & 2307,7 & 200,0 & 40,0 & 4,0 & 32,0 \\
$\mathbf{1 0}$ & 2307,7 & 200,0 & 40,0 & 7,0 & 14,0 \\
$\mathbf{1 1}$ & 2307,7 & 200,0 & 80,0 & 4,0 & 19,0 \\
$\mathbf{1 2}$ & 2307,7 & 200,0 & 80,0 & 7,0 & 36,0 \\
$\mathbf{1 3}$ & 2307,7 & 300,0 & 40,0 & 4,0 & 33,0 \\
$\mathbf{1 4}$ & 2307,7 & 300,0 & 40,0 & 7,0 & 13,0 \\
$\mathbf{1 5}$ & 2307,7 & 300,0 & 80,0 & 4,0 & 33,0 \\
$\mathbf{1 6}$ & 2307,7 & 300,0 & 80,0 & 7,0 & 34,0 \\
$\mathbf{1 7}$ & 576,9 & 250,0 & 60,0 & 5,0 & 22,0 \\
$\mathbf{1 8}$ & 2884,6 & 250,0 & 60,0 & 5,5 & 42,0 \\
$\mathbf{1 9}$ & 1730,8 & 150,0 & 60,0 & 5,5 & 23,0 \\
$\mathbf{2 0}$ & 1730,8 & 350,0 & 60,0 & 5,5 & 38,0 \\
$\mathbf{2 1}$ & 1730,8 & 250,0 & 20,0 & 5,5 & 34,0 \\
$\mathbf{2 2}$ & 1730,8 & 250,0 & 100,0 & 5,0 & 33,0 \\
$\mathbf{2 3}$ & 1730,8 & 250,0 & 60,0 & 2,5 & 26,0 \\
$\mathbf{2 4}$ & 1730,8 & 250,0 & 60,0 & 8,5 & 26,0 \\
$\mathbf{2 5}$ & 1730,8 & 250,0 & 60,0 & 5,5 & 39,0 \\
$\mathbf{2 6}$ & 1730,8 & 250,0 & 60,0 & 5,5 & 38,0 \\
$\mathbf{2 7}$ & 1730,8 & 250,0 & 60,0 & 5,5 & 40,0 \\
\hline
\end{tabular}

As enzimas peroxidases catalisam uma variedade de reações, porém todas requerem a presença de peróxido de hidrogênio para ativar a enzima. Vários estudos relatam a influências de fatores como peróxido de hidrogênio, concentração de corante e enzima. Silva et al., (2012) realizaram a descoloração dos corantes azo remazol laranja brilhante 3R e vermelho remazol ultra, foram obtidas degradações de $9 \%$ e 5,5\% respectivamente, esta baixa descoloração pode estar relacionada a fatores como concentração de enzima, peróxido de hidrogênio ou concentração de corante.

Yousefi e Hamid-Reza (2010) estudaram a degradação do corante ácido laranja 7 por peroxidase do fungo Coprinus cinereus, sendo determinados os principais fatores que influenciam na reação enzimática, entre estes também foi identificada a concentração de peróxido de hidrogênio. Segundo Yousefi e Hamid-Reza (2010) a reação não ocorre sem adição de peróxido de hidrogênio e a taxa de descoloração inicial aumentou à medida que a concentração de peróxido de hidrogênio aumentou de 1,3 $\mathrm{mM}$ a 2,6 mM, mas diminui em maiores concentrações. 
Na tabela 3 podem-se observar os efeitos das variáveis sobre a descoloração do corante. Na Figura 2 é apresentada a curva de nível da interação peróxido de hidrogênio $\left(\mathrm{H}_{2} \mathrm{O}_{2}\right)$ e $\mathrm{pH}$.

Tabela 3- Efeitos das variáveis sobre a descoloração do corante

\begin{tabular}{ccccccc}
\hline Fator & Efeito & $\begin{array}{c}\text { Erro } \\
\text { padrão }\end{array}$ & t student & $\mathbf{p}$ & \multicolumn{2}{c}{ Limite de confiança } \\
\cline { 6 - 7 } & & & & $\mathbf{- 9 5 \%}$ & $\mathbf{+ 9 5 \%}$ \\
\hline Média/interações & $\mathbf{3 9 , 0 0 0}$ & $\mathbf{4 , 5 9 9}$ & $\mathbf{8 , 4 7 8}$ & $\mathbf{0 , 0 0 0}$ & $\mathbf{2 8 , 9 7 7}$ & $\mathbf{4 9 , 0 2 2}$ \\
(1)Enzima (L) & 5,749 & 3,252 & 1,767 & 0,102 & $-1,337$ & 12,837 \\
Enzima (Q) & $-5,354$ & 3,449 & $-1,551$ & 0,147 & $-12,871$ & 2,162 \\
$(\mathbf{2}) \mathbf{H}_{2} \mathbf{O}_{2}$ (L) & $\mathbf{7 , 9 1 6}$ & $\mathbf{3 , 2 5 2}$ & $\mathbf{0 , 0 3 1 5}$ & $\mathbf{0 , 0 3 1}$ & $\mathbf{0 , 8 3 0}$ & $\mathbf{1 5 , 0 0 4}$ \\
$\mathrm{H}_{2} \mathrm{O}_{2}$ (Q) & $-6,104$ & 3,449 & 0,102 & 0,102 & $-13,621$ & 1,413 \\
(3) Corante (L) & 2,916 & 3,252 & 0,388 & 0,388 & $-4,170$ & 10,004 \\
Corante (Q) & $-4,604$ & 3,449 & 0,206 & 0,207 & $-12,121$ & 2,913 \\
(4) pH (L) & $-2,250$ & 3,252 & 0,502 & 0,502 & $-9,337$ & 4,837 \\
pH (Q) & $\mathbf{- 8 , 3 5 4}$ & $\mathbf{3 , 4 4 9}$ & $\mathbf{0 , 0 3 2 2}$ & $\mathbf{0 , 0 3 2}$ & $\mathbf{- 1 5 , 8 7 1}$ & $\mathbf{- 0 , 8 3 7}$ \\
1L e 2L & $-5,125$ & 3,983 & 0,222 & 0,222 & $-13,804$ & 3,554 \\
1L e 3L & 2,875 & 3,983 & 0,484 & 0,484 & $-5,805$ & 11,554 \\
1L e 4L & $-1,625$ & 3,983 & 0,690 & 0,690 & $-10,304$ & 7,054 \\
2L e 3L & 3,375 & 3,983 & 0,413 & 0,413 & $-5,304$ & 12,055 \\
2 L e 4 L & 4,875 & 3,983 & 0,244 & 0,244 & $-3,804$ & 13,554 \\
3L e 4L & $\mathbf{9 , 8 7 5}$ & $\mathbf{3 , 9 8 3}$ & $\mathbf{0 , 0 2 9}$ & $\mathbf{0 , 0 2 9}$ & $\mathbf{1 , 1 9 5}$ & $\mathbf{1 8 , 5 5 4}$ \\
\hline
\end{tabular}

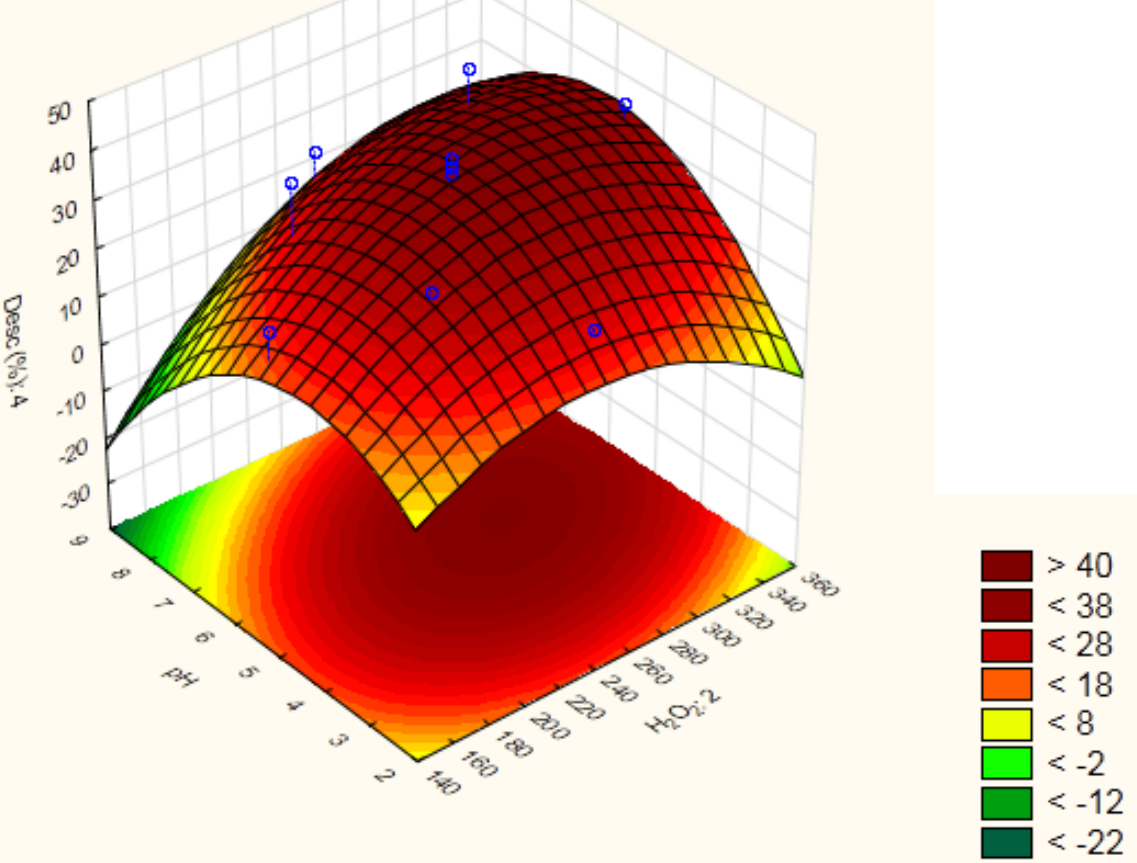

Figura 2- Curva de nível da descoloração de corante em função de pH e concentração de Peróxido. 
A Figura 2 representa o efeito de diferentes concentrações de $\mathrm{H}_{2} \mathrm{O}_{2}$ e $\mathrm{pH}$. As maiores descolorações do corante de urucum foram obtidas numa faixa de concentração de peróxido de hidrogênio entre 240 e 330 e uma faixa de pH entre 4,5 e 6,5. Além do peróxido de hidrogênio o pH também foi um fator significativo na reação. A máxima descoloração foi obtida em $\mathrm{pH} 7,0$ porém em $\mathrm{pH} 5,5$ também foi obtida uma degradação de até $42 \%$. A interação do corante com $\mathrm{pH}$ também se mostrou um fator significativo para a reação. Testes preliminares mostraram que a absorbância das soluções de corante em varia com de acordo com pH. Para um limite de confiança de $95 \%$ foi obtido um ajuste ao modelo de $70 \%$. O modelo para a descoloração do corante de urucum é representado na equação 3.

$\mathrm{y}=39,00+3,96\left[\mathrm{H}_{2} \mathrm{O}_{2}\right]-4,17[\mathrm{pH}]^{2}+4,94[\mathrm{pH}] .[$ corante $]$

Toda enzima tem um $\mathrm{pH}$ característico no qual sua atividade é máxima. Com a mudança do $\mathrm{pH}$ as interações eletrostáticas dentro da enzima se alteram com a possibilidade de alterar a conformação tridimensional e assim sua atividade. De acordo com Cavaco e Gubits, 2003, quando o substrato se liga a enzima, esse também é influenciado pelas interações.

A maioria das enzimas tem um intervalo de tolerância determinado de $\mathrm{pH}$, muitas vezes maior do que uma unidade de $\mathrm{pH}$, em que sua atividade é de 80 a $100 \%$ do seu máximo. Dentro desse intervalo, o pH pode mudar sem consequências importantes para sua actividade. Mohan; Prasad; Sarma, (2005) avaliaram a descoloração do corante ácido Preto $10 \mathrm{BX}$, variando o pH de 2 a 9, a máxima degradação foi obtida em $\mathrm{pH}$ 2, 67\%. Em pH acima de 2, o $\mathrm{pH}$ caiu significativamente.

\section{CONCLUSÃO}

A descoloração do corante de urucum pela enzima Horseradish peroxidase é influenciada pela concentração do peróxido de hidrogénio e pelo $\mathrm{pH}$ do meio. $\mathrm{O}$ corante de urucum foi degradado pela enzima com descoloração máxima de $44 \%$. O uso da enzima Horseradish peroxidase como rota para a degradação do corante natural de urucum torna este processo de tingimento mais sustentável sob o aspecto ambiental. O uso de pool enzimático pode ser uma alternativa para a melhoria da eficiência do processo.

\section{REFERÊNCIAS}

BALAN, K.; SATHISHKUMAR, P.; PALVANNAN, T. Decolorization of malachite green by laccase: Optimization by response surface methodology. J. Taiwan Inst. Chem. Eng., v. 43, p. 776-782, 2012.

BUCHANAN, I. D.; NICELL, J. A. Kinetics of Peroxidase Interactions in the Presence of a Protective Additive. J. Chem. Technol. Biotechnol., v. 72, p. 23-32, 1998.

CAVACO, P. A.; GUBITS, G.M. Textile processing with enzymes, Ed CRC, p.163, 2003 
HADDAR, W.; TICHA, M. B.; GUESMI, A.; KHOFLI, F.; DURAND, B. A novel approach for a natural dyeing process of cotton fabric with Hibiscus mutabilis (Gulzuba): process development and optimization using statistical analysis. J. Cleaner Prod., v. 68, p. 114-120, 2014.

KARAM, J.; NICELL, J. Potential applications of enzymes in waste treatment. J. Chem. Tech. Biotechnol., v. 69, p. 141-153, 1997.

KARIM, Z.; ADNAN, R.; HUSAIN, Q. A $\beta$-cyclodextrin-chitosan complex as the immobilization matrix for horseradish peroxidase and its application for the removal of azo dyes from textile effluent. Int. Biodeter. Biodeg., v. 72, p. 10-17, 2012.

KUMAR, V. V.; SATHYASELVABALA, V.; PREMKUMAR, M. P.; VIDYADEVI, T.; SIVANESAN, S. Biochemical characterization of three phase partitioned laccase and its application in decolorization and degradation of synthetic dyes. J. Mol. Catal. B: Enzym., v. 74, p. 63-72, 2012.

MOHAN, S. V.; PRASAD, K. K.; RAO, N. C.; SARMA P. N. Acid azo dye degradation by free and immobilized Horseradish peroxidase (HRP) catalyzed process. Chemosphere, v. 58, p. 1097-1105, 2005.

MOHAMMAD, S.; UL-ISLAM, S.; MOHAMMAD, F. Recent advancements in natural dye applications: a review. J. Cleaner Prod., v. 53, p. 310-331, 2013.

NGUYEN, T. A.; JUANG, R-S. Treatment of waters and wastewaters containing sulfur dyes: A review. Chem. Eng. J.. v. 219, p. 109-117, 2013.

SILVA, M. C.; CORREA, A. D.; TORRES, J. A.; AMORIM, M. T. S. P. Descoloração de corantes industriais e efluentes têxteis simulados por peroxidase de nabo (Brassica campestre). Quím. Nova, v. 35, n. 5, p. 889-894, 2012.

SILVA, M. C.; TORRES, J. A.; SÁ, L. R. V.; P; CHAGAS, P. M. B.; FERREIRA-LEITÃO, V. S.; CORREA, A. D. The use of soybean peroxidase in the decolourization of Remazol Brilliant Blue R and toxicological evaluation of its degradation products. J. Mol. Catal. B: Enzym., v. 89, p. 122-129, 2013.

TAVARES, A. P. M.; CRISTÓVÃO, R. O.; LOUREIRO, J. M.; BOAVENTURA, R. A. R.; MACEDO, E. A. Application of statistical experimental methodology to optimize reactive dye decolourization by commercial laccase. J. Hazard. Mater., v. 162 p. 1255-1260, 2009.

YOUSEFI, V.; HAMID-REZA, K. Statistical analysis for enzymatic decolorization of acid orange. Int. Biodeter. Biodeg., v. 64, p. 245-252, 2010.

WU, Y.; TAYLOR, K. E.; BISWAS, N.; BEWTRA, J. K. Comparison of Additives in the Removal of Phenolic Compounds by Peroxidase Catalyzed Polymerization. Water Res., v. 31, p. 2699-2704, 199 